Introduction In Myanmar, People Who Inject Drugs (PWIDs) has the highest HIV prevalence of $18.7 \%$ in comparing to other key population. Establishment of drop-in-centre (DIC) and provision of services has been proved as successful intervention for key population. An assessment was conducted to determine the knowledge on sexually transmitted infections (STI)/HIV and utilisation of drop-in-centres among drug users after the establishment of DIC and out-reach activities.

Methods A community-based, post-intervention assessment was conducted using quantitative and qualitative methods in underserved areas of north-western border in Myanmar.

Results A total of 202 drug users, 156 PWIDs and 46 People Who Use Drugs (PWUDs), were included in the study. Their age ranged from ranged from 18 to 60 years with the mean age of $34.9 \pm 8.2$ years. Regarding STI knowledge, no one sought to treat STD at the beginning of the intervention but $4 \%$ of drug users searched for the treatment at the end. Over $25 \%$ knew at least one symptom of STI in men while only $15.3 \%$ recognised at least one symptom in women. Knowledge on higher risk of blood borne infections such as Hepatitis B $(25 \%$ vs. $51 \%, \mathrm{p}<0.1)$, Hepatitis C $(8 \%$ vs. $13 \%$, $\mathrm{p}<0.05)$ was improved in comparing to pre-intervention. HIV testing rate within six months was also increased from $10 \%$ to $74 \%(\mathrm{p}<0.001)$. Regarding sexual behaviours, percent of drug users who had sex with paid sex partner and who had more than one sexual partner within 6 months were significantly reduced $(11 \%$ vs. $0.5 \%, \mathrm{p}<0.05 ; 11 \%$ vs. $1 \%$, $\mathrm{p}<0.05)$. Regarding drop in centre (DIC), more drug users were aware of DIC $(54 \%$ vs. $91 \%, \mathrm{p}<0.01)$ and ever visited there $(22 \%$ vs. $76 \%, p<0.01)$. Almost all of them $(94 \%)$ ever received DIC services consisted of HIV testing and counselling $(47 \%)$ and health education service (68\%). Nearly half of them (46\%) received outreach services and mobile HIV testing covered about $16 \%$.

Conclusion Improvement in knowledge and behaviour regarding STI/HIV and DIC utilisation were seen among the drug users after the intervention.

\section{P3.126 COUNTY-LEVEL SOCIODEMOGRAPHIC FACTORS ASSOCIATED WITH REPORTED CONGENITAL SYPHILIS IN UNITED STATES 2012-2015}

Kyle Bernstein, Kendra Cuffe, Sarah Kidd, Virginia Bowen, Elizabeth Torrone, Jami Leichliter. CDC - Atlanta, USA

\subsection{6/sextrans-2017-053264.361}

Introduction Pregnant women - In the US, Congenital Syphilis (CS) rates have increased 48\% between 2012 and 2015. We explored county level sociodemographic factors associated with having reported at least one CS case during 2012 and 2015 among all US counties.

Methods The $321(10.2 \%)$ US counties that reported at least 1 CS case during 2012-2015 were compared to the remaining $2829(89.8 \%)$ US counties with no reported CS. Multivariable logistic regression models were constructed to assess factors independently associated with counties reporting CS using adjusted Odds Ratios (aOR). County level factors examined included: $\%$ population black [above or below the national median],\% Hispanic population [above or below the national median], Medicaid expansion state, violent crime rate per 100,000 , and the $\%$ change in adult male and female syphilis cases between during 2012 and 2015. Final models were determined by comparing likelihood ratio statistics.

Results Independent county level factors associated with reporting at least one CS case during 2012-2015 were: Medicaid expansion state $[\mathrm{aOR}=2.24,95 \%$ CI $1.51-3.34], \%$ black population $[\mathrm{aOR}=2.86,95 \%$ CI $1.79-4.57], \%$ Hispanic $[\mathrm{aOR}=2.13,95 \% \mathrm{CI} 1.41-3.24], 10$ unit change in violent crime rate $[\mathrm{aOR}=1.04,95 \% \mathrm{CI} 1.02-1.05]$, and 10 unit change in $\%$ change in female syphilis $[\mathrm{aOR}=1.02,95 \% \mathrm{CI}$ 1.00-1.03].

Conclusion CS in the US is highly geographically focused with only $10.2 \%$ of counties reporting any cases in the past 4 years. Socioeconomic and demographic factors working at the community level are associated with increased odds of having reported a case of CS. Interventions to prevent CS in the US should focus at community, as well as individual level.

\section{P3.127 ANALYSIS ON ACCURATE DIAGNOSIS AND STANDARDISED TREATMENT OF REPORTED SYPHILIS CASES IN SHENZHEN}

Lan Li-Na, Wu Xiao-Bing, Zhang Chun-Lai, Wen Li-Zhang, Tang Fen, Hong Fu-Chang. Shenzhen Centre for Chronic Disease Control, Shenzhen - China Popular Republic

\subsection{6/sextrans-2017-053264.362}

Introduction To investigate the accurate rate of diagnosis and the standardised rate of treatment of reported syphilis cases in Shenzhen and to provide evidence for further prevention strategies.

Methods Data of syphilis cases reported from July 1, 2013 to June 30, 2014 were downloaded from the 'China Information System for Disease Control and Prevertion'. Randomised sampling method was used to select cases from the download database. Medical staff compared the information from the selected cases with doctors' daily record, patients' laboratory test results and pharmacy prescriptions from medical institutions. The accuracy of diagnosis and standardisation of treatment were evaluated based on the Diagnostic Criteria and Management of Syphilis Guidelines (Ministry of Health, China, 2007).

Results This study totally selected 1589 syphilis cases for evaluation. The accurate rate of diagnosis was $95.53 \%$ for total, and the rates were $83.59 \%, 96.60 \%, 97.73 \%, 100.00 \%$ and $97.37 \%$ respectively for primary, secondary, tertiary, congenital and latent syphilis. Nearly $66.08 \%$ cases were treated with penicillin therapy and $1.76 \%$ cases were treated with replacement therapy. The standardised rate of treatment was $67.84 \%$. Conclusion The accurate rate of syphilis diagnosis is high in Shenzhen, however, the standardised rate of treatment is far from the goal set in the national syphilis control plan. It suggests that training and supervision should be enhanced and modification of communicable disease card (such as adding the diagnosis and treatment information of syphilis cases) should be considered, to improve the rate of accurate diagnosis and standardised treatment of syphilis cases. 
P3.128 DEVELOPMENT OF A RISK CALCULATOR FOR THE 3MONTH PREDICTION OF INCIDENT SYPHILIS INFECTION AMONG HIGH-RISK MEN WHO HAVE SEX WITH MEN AND TRANSGENDER WOMEN PRESENTING TO A STD CLINIC IN LIMA, PERU

${ }^{1}$ Lao-Tzu Allan-Blitz, ${ }^{2}$ Kelika A Konda, ${ }^{3}$ Silver K Vargas, ${ }^{3}$ Eddy R Segura, ${ }^{3}$ Gino M Calvo, ${ }^{3}$ Carlos F Caceres, ${ }^{2}$ Jeffrey D Klausner. ${ }^{1}$ David Geffen School of Medicine, UCLA, And Saphir, Los Angeles, USA; 'Department of Medicine, David Geffen School of Medicine, University of California Los Angeles Los Angeles, USA; ${ }^{3}$ Universidad Peruana Cayetano Heredia, Lima - Peru

10.1136/sextrans-2017-053264.363

Introduction Syphilis incidence worldwide has rebounded since 2000, especially among men who have sex with men (MSM). A predictive model for incident syphilis infection may inform prevention counselling and use of chemoprophylaxis.

Methods We analysed data from a longitudinal study of a STD clinic-based cohort of MSM and transgender women reporting a history of HIV or syphilis infection and/or highrisk sexual behaviour, followed quarterly for two years. Incident infection was defined as a four-fold increase in RPR titers or new RPR reactivity if two prior titers were non-reactive. We used generalised estimating equations with a Poisson regression to develop a predictive model of syphilis incidence in one-half of the data set, and verified the model in the second half, calculating an area under the curve (AUC), summarising specificity and sensitivity. We then applied the final model to the full baseline dataset. Finally we developed an online risk calculator from our model.

Results Among 401 participants enrolled, 22\% were transgender women and $31 \%$ were HIV-infected at baseline. Syphilis incidence was 19.9 cases per 100-person years (95\% CI 16.324.3). Predictors of syphilis incidence were HIV infection, high number of male sex partners (categorised as: $0,1,2-3$, $4-9,>10)$, history of syphilis infection, receptive and versatile anal sex role and condomless receptive anal sex. The AUC was $71 \%(95 \%$ CI $64 \%-78 \%)$ in the validation dataset for incident syphilis infection in the next 3 months. Those at highest risk had a 1-in-7 likelihood of syphilis infection in the next 3 months. When applied to the baseline dataset the AUC was $71 \%$ (95\% CI 65\%-77\%) for predicting recent syphilis infection. The online syphilis risk calculator is available at: www.syphrisk.net (English), www.sifriesgo.net (Spanish).

Conclusion Our results show that the likelihood of syphilis infection among a high-risk STD clinic-based cohort can be estimated accurately. Our calculator may guide STD clinical management by directing risk behaviour counselling and potential use of doxycycline chemoprophylaxis.

\section{P3.129 HOW CAN WE USE PHYLOGENETICS TO FACILITATE CLINICAL CASE FINDING AND PARTNER NOTIFICATION IN HIV: LESSONS FROM A SYSTEMATIC REVIEW OF ITS USE IN STIGMATISED INFECTIOUS DISEASES}

${ }^{1}$ Larissa Mulka, 'Jaime H Vera, ${ }^{2}$ Andrew J Leigh-Brown, ${ }^{1}$ Jackie A Cassell. ${ }^{1}$ Brighton and Sussex Medical School, Brighton, UK; ${ }^{2}$ University of Edinburgh, Edinburgh, UK

\subsection{6/sextrans-2017-053264.364}

Introduction Phylogenetic information provides new horizons for clinical case finding in HIV, but raises issues of acceptability, privacy and even criminalisation. We reviewed studies describing use of phylogenetics to directly inform case finding in community acquired stigmatised infectious diseases.

Methods A search in MEDLINE, Embase, CINAHL and PsychINFO for articles where phylogenetics have been used to facilitate case finding in sexually transmitted infections, TB, HBV or HCV, published until July 2016 in English.

Results 26 of 6042 papers screened met the inclusion criteria; 17 TB, 9 HIV. 19 studies reported using phylogenetics to identify and investigate HIV outbreaks but did not report its role in case finding. Case finding strategies included confirming the source of an outbreak to prompt wider investigation (HIV); investigation of phylogenetically clustered cases (TB, HIV); combined cluster and geographical information to target screening (TB); screening informed by discrepancies between genotypic and epidemiological data (TB); phylogenetic characterisation to inform a screening intervention (HIV); epidemiological data to identify of a source (HIV); and contact tracing with genotype matching to a phenotype (HIV). Facilitators included sharing molecular surveillance data to establish community support in targeted TB screening. Barriers included delayed results, time lapse between cases and refusal of access to premises for screening. However patient barriers were rarely reported. Ethical issues included media coverage of an HIV sources identity.

Conclusion Phylogenetics-informed approaches to case finding are feasible in stigmatised infections. However studies reporting their use in clinical and public health practice provide limited information on patient related barriers, acceptability, or on ethical challenges such as identification of "core" transmitters or criminalisation. Research into patient views on acceptability, risks and preferred approaches to using phylogenetic information for case finding in HIV is needed to inform interventions.

\section{P3.130 POTENTIAL IMPACT OF TESTING FOR MYCOPLASMA GENITALIUM INFECTION AND MACROLIDE RESISTANCE: A MATHEMATICAL MODELLING ANALYSIS}

${ }^{1}$ Laura C Chambers, ${ }^{2}$ Lisa E Manhart, ${ }^{1}$ Rachel A Silverman, ${ }^{3}$ Ruanne V Barnabas. ${ }^{1}$ University of Washington, Department of Epidemiology, Seattle, USA; ${ }^{2}$ University of Washington, Departments of Epidemiology and Global Health, Seattle, USA; ${ }^{3}$ University of Washington, Departments of Medicine, Global Health, and Epidemiology, Seattle, USA

\subsection{6/sextrans-2017-053264.365}

Introduction Patients with genitourinary symptoms are generally treated syndromically with azithromycin, which can induce macrolide resistance in Mycoplasma genitalium (MG). Directing treatment based on aetiology and known macrolide susceptibility may prevent emergence of resistance. We constructed a mathematical model to evaluate the potential impact of simultaneous detection of $\mathrm{MG}$ and resistance markers on the percent of MG infections that are macrolidesusceptible.

Methods We developed a gender- and risk-stratified, compartmental model of MG transmission within a heterosexual population. We assumed clearance of untreated infections in 30 days; development of symptoms in $2.4 \%$ of infected men and $5.1 \%$ of infected women; initial treatment of symptomatic men and women with azithromycin; treatment of men with persistent/recurrent symptoms with moxifloxacin; 50\% of infections macrolide-susceptible at baseline; de-novo macrolide resistance in $18 \%$ of susceptible bacteria after azithromycin therapy; and 100\% efficacy of moxifloxacin. The model was 